\title{
LOCAIS DE IMPLANTE DE MICROCHIPS DE IDENTIFICAÇÃO ELETRÔNICA DE LEITÕES: SELEÇÃO E VALIDAÇÃO POR ANÁLISE DE IMAGEM
}

\author{
HÉLITON PANDORFI ${ }^{1}$, IRAN J. O. SILVA ${ }^{2}$, KELLY B. SEVEGNANI ${ }^{3}$, IVAN W. CARO ${ }^{4}$
}

RESUMO: Nas últimas décadas, a produção de suínos, pressionada por uma crescente demanda por alimentos, tem-se caracterizado pela maior concentração de animais em grandes unidades de produção, dificultando o registro dos dados individuais. Os sistemas automáticos de identificação eletrônica podem auxiliar a detecção de doenças, a avaliação de respostas fisiológicas, o controle de ingestão de alimentos, a atividade física e ainda o impacto ambiental causado pelo sistema de produção, promovendo melhor controle da propriedade. Transponders injetáveis, brincos eletrônicos e o monitoramento por meio da análise de imagem estão sendo utilizados no processo de identificação. $\mathrm{O}$ objetivo desta pesquisa foi avaliar os diferentes locais de implante subcutâneo de microchips em leitões, verificando-se possíveis infecções e/ou rejeições, migrações dos microchips em relação ao local de implante e sua validação em relação à análise de imagem.

PALAVRAS-CHAVE: análise de imagem, identificação eletrônica, zootecnia de precisão.

\section{POINTS OF MICROCHIPS IMPLANTS IN PIGS FOR ELETRONIC IDENTIFICATION: PLACING AND VALIDATION BY IMAGE ANALYSIS}

\begin{abstract}
In the last decades the increase of the swine production, because of the increasing in food consumption, has been characterized by an increase of animal concentration in great production units, making difficult to record individual data. The automatic electronic identification systems can help in illness detection and in the evaluation of physiological data, food ingestion, physical activity and impact in the environment by the production system. Electronic injectable transponders, earing tags and the image analysis are been used for electronic identification purposes. The objective of the present work was to evaluate the best body site to inject passive transponders in piglets and possible resulting infections and/or rejections and its validation by using image analysis.
\end{abstract}

KEYWORDS: image analysis, electronic identification, animal precision production.

\section{INTRODUÇÃO}

A preocupação com a produção de alimentos está cada vez mais voltada para a quantidade e a qualidade do produto final e as suas implicações com o meio ambiente. Isso está acontecendo justamente no momento em que novas tecnologias de controle surgem, novas exigências de mercado aparecem, restrições de consumo e produção crescem diariamente, em paralelo com a mudança de hábitos da população, a qual vem exigindo produtos finais de melhor qualidade. Por outro lado, a competitividade interna e externa está acirrada, visando à ocupação de espaço seguro no mercado.

A União Européia preconiza a identificação de todos os animais (bovinos, suínos, caprinos e ovinos) para total controle do nascimento à linha de abate, definindo um padrão mundial para os

\footnotetext{
${ }^{1}$ Eng ${ }^{0}$ Agrônomo, Doutorando do Curso de Física do Ambiente Agrícola, NUPEA/ESALQ-USP. Departamento de Ciências Exatas, Av. Pádua Dias, 11, Caixa Postal 9, Piracicaba - SP, Fone: (0XX19) 3429.4217, Ramal 241, hpandorf@esalq.usp.br

${ }^{2}$ Eng $^{\circ}$ Agrícola, Prof. Doutor, Departamento de Engenharia Rural, NUPEA/ESALQ-USP, Piracicaba - SP.

${ }^{3}$ Eng ${ }^{\circ}$ Agrônomo, Profa. Doutora, UD Registro - UNESP, Registro - SP.

${ }^{4}$ Eng ${ }^{\mathrm{o}}$ Automação, NUPEA/ESALQ-USP.

Recebido pelo Conselho Editorial em: 24-6-2003
}

Aprovado pelo Conselho Editorial em: 28-10-2004 
dispositivos eletrônicos de identificação e assegurando a compatibilidade entre os sistemas dos diferentes fabricantes, possibilitando dessa maneira a ampla projeção do registro e identificação de todos os animais (WISMANS, 1999). Assuntos como rastreabilidade da produção, selo de qualidade e zootecnia de precisão estão sendo discutidos em todas as cadeias produtivas, com o objetivo de maximizar os sistemas existentes em prol de melhorias nas condições de produção e, conseqüentemente, do produto final, visando à segurança alimentar.

A utilização da tecnologia de identificação eletrônica (ID) de animais no Brasil encontra-se numa fase inicial e somente algumas empresas agropecuárias estão fazendo uso da técnica de implantação de microchips e leitura por meio de leitor de rádio-freqüência (CURTO et al., 1997). As expectativas com a utilização desses microchips baseiam-se na grande quantidade de informações comportamentais que podem ser obtidas por meio de monitoramento digital diário, auxiliado por um sistema informatizado, possibilitando melhor análise do bem-estar animal e a possibilidade de gerar novas tecnologias na área de produção animal.

Dessa forma, não se pode mais admitir que se fale de sistema de produção animal ou de cadeia produtiva, sem se considerar esses novos conceitos. Diante disso, a utilização de novas tecnologias e ferramentas, como a informática (softwares), microeletrônica, análise de imagem, sensores e atuadores, podem melhorar o trabalho experimental, favorecendo uma acurácia das pesquisas até então não alcançada pelos métodos tradicionais de observação.

Seja qual for o dispositivo, um identificador eletrônico possui um transmissor/receptor que emite um número de série que permite identificar o animal e monitorar a sua atividade. A sua antena cria um campo eletromagnético, que é usado pelo transponder para gerar a própria voltagem operacional como uma fração de frequiência de ativação, emitindo por sua vez um sinal que retorna ao transceptor e comparando o número do animal com uma base de dados que contêm registrados todos os animais da granja (ARTMANN, 1999).

ARTMANN (1999) salientou que o local de aplicação dos transponders deve ser de fácil acesso, permitindo maior eficiência no processo de identificação e remoção, de modo que não haja danos na carcaça. Para suínos, em geral, o autor sugere que a região da cabeça talvez seja o local mais indicado para transponders injetados debaixo da pele.

Entretanto, a injeção subcutânea de um transponder causa danos a pequenas partes da epiderme e derme, cuja cura subseqüente pode ser normal ou transtornada. Além disso, diversas reações no corpo do animal poderiam ser resultado de movimentos migratórios do microchip (ARNDT \& WIEDEMANN, 1991).

Embora a pele seja constituída por uma complexa estrutura de fibras musculares, tecido conjuntivo, folículos de pêlo, glândulas e vasos sangüíneos, MAIBACH \& ROVEE (1972) comentam que a cura das feridas causadas pelos implantes é caracterizada por uma quase perfeita regeneração da epiderme em aproximadamente uma semana, e a derme em aproximadamente três semanas. Com base nessa experiência, espera-se que a cura da ferida após a injeção de um transponder seja bastante rápida (LAMBOOY, 1990).

ARNDT \& WIEDEMANN (1991) concluíram que microchips aplicados de maneira correta e com total assepsia não apresentavam infecção ou rejeição, sendo uma das principais razões para o bom resultado o fato de os transponders injetáveis serem encapsulados dentro de um tubo de vidro biocompatível, que separa o implante do tecido anfitrião, permanecendo sem sofrer nenhuma alteração durante todo o período de vida do animal.

As migrações são aspectos críticos para o uso de transponders, podendo ser um risco para alguns órgãos essenciais, além de possíveis dificuldades na recuperação dos microchips por ocasião do abate, quando os mesmos não estão localizados no local esperado (LAMBOOY, 1990). 
Para avaliar a mudança da distância entre um transponder e o local de aplicação, são utilizadas várias metodologias, tais como radiografias e dissecação do cadáver para posterior cálculo da distância de migração, usando-se pontos de referência no corpo, sendo bastante difícil comparar os poucos resultados disponíveis e os diferentes locais de implante usados em diferentes espécies (LAMBOOY \& MERKS, 1989).

Por exemplo, LAMBOOY \& MERKS (1989) conduziram um experimento em que foram implantados transponders na parte de trás da orelha em 11 leitões. Posteriormente, na linha de abate, só foram encontrados três dos transponders no local original de injeção, sendo quatro localizados na mandíbula e alguns achados até mesmo próximos da coluna espinal.

Fatores como idade dos animais e tamanho dos transponders têm influência significativa na migração. Com o aumento da idade dos animais e o tamanho dos implantes, há diminuição dos movimentos observados, embora não se possa confirmar uma interação positiva entre ambos os fatores (KERN, 1997). Apesar de esses fatores de influência terem sido mencionados, o autor afirmou que migrações de, no máximo, $60 \mathrm{~mm}$ podem ser assumidas como não-críticas, pois o transponder ainda está na região próxima à inserção, esperando-se que esse seja localizado sob condições normais por ocasião do abate.

O objetivo desta pesquisa foi avaliar os diferentes locais de implante subcutâneo de microchip em leitões, verificando infecções e/ou rejeições, possíveis migrações dos microchips em relação ao local de implante e sua validação por meio da análise de imagem.

\section{MATERIAL E MÉTODOS}

O experimento foi realizado nas dependências do Departamento de Produção Animal, Setor de Não-Ruminantes, da Universidade de São Paulo, Câmpus de Piracicaba da Escola Superior de Agricultura "Luiz de Queiroz". Foram utilizados nove leitões da raça Landrace, que ficaram alojados na maternidade, em cela parideira, durante o período de estudo. $\mathrm{O}$ experimento foi iniciado com o implante dos microchips no terceiro dia após o nascimento dos animais, com peso médio de 1,557 kg. Os microchips implantados foram da marca comercial Destron Fearing, apresentando dimensões 2,2 x 11,5 mm e encapsulados em vidro biocompatível. O microchip é um componente passivo, ou seja, apenas retransmite um sinal, sem necessidade de uma fonte de alimentação e que pode ser captado por uma antena. Por meio de um protocolo de comunicação, a informação transmitida permite o registro dos dados na unidade de memória e posteriormente a alimentação de um banco de dados.

Os tratamentos correspondentes aos diferentes locais de implante foram: 1) entre os dígitos da pata; 2) na cartilagem na base da orelha, e 3) na fossa infra-orbital, perfazendo total de três tratamentos com três repetições cada.

Os implantes foram realizados com auxílio de um injetor com agulha oca, sendo injetados subcutaneamente em diferentes posições no corpo do leitão. As inserções foram feitas após desinfecção do local de aplicação com algodão embebido em álcool, além do uso de luvas descartáveis pelo aplicador, garantindo total assepsia no momento do implante. A aplicação foi realizada sempre com o auxílio de duas pessoas - uma para a imobilização do animal e a outra na aplicação propriamente dita. Após o implante dos microchips, os leitões permaneciam na maternidade sob as mesmas condições ambientais e nutricionais, durante todo o experimento.

Para avaliar as alterações da distância entre um transponder e o local de aplicação, foram realizadas sessões de radiografias no dia da operação, $4^{\underline{0}}$ e $11^{\underline{0}}$ dia após o mesmo, utilizando posteriormente as radiografias para o cálculo da distância de migração, considerando pontos de referência corporais. Partindo de um ponto inicial, traçava-se um eixo e determinavam-se as coordenadas cartesianas para a localização de cada microchip, considerando-se o ponto inicial como 
zero. Os valores foram medidos em milímetros $(\mathrm{mm})$, sendo no final somados para a série de três radiografias, determinando-se a distância da migração no dia do implante, no $4^{\underline{0}}$ e $11^{\circ}$ dias após o implante, por meio das seguintes equações:

$$
\begin{array}{ll}
1^{\text {a }} \text { Avaliação } & \mathrm{d}(\mathrm{AB})=\sqrt{(\mathrm{XB}-\mathrm{XA})^{2}+(\mathrm{YB}-\mathrm{YA})^{2}} \\
2^{\text {a }} \text { Avaliação } & \mathrm{d}(\mathrm{BC})=\sqrt{(\mathrm{XC}-\mathrm{XB})^{2}+(\mathrm{YC}-\mathrm{YB})^{2}} \\
3^{\mathrm{a}} \text { Avaliação } & \mathrm{d}(\mathrm{CD})=\sqrt{(\mathrm{XD}-\mathrm{XC})^{2}+(\mathrm{YD}-\mathrm{YC})^{2}}
\end{array}
$$

Após a determinação do melhor local de implante, a eficiência do sistema de identificação eletrônica foi avaliada, utilizando-se como referência o monitoramento da atividade dos animais por meio de microcâmeras e posterior análise das imagens.

O ponto fundamental desta pesquisa foi justamente a avaliação dos sistemas de identificação eletrônica, usando a análise comportamental dos leitões, para compreensão da eficiência dos sistemas em função da atividade dos animais. Para tanto, usou-se como ferramenta de registro a identificação eletrônica por imagem, por meio de microcâmeras instaladas no interior do abrigo escamoteador. Foram utilizadas microcâmeras preto e branco, com lente de $2,45 \mathrm{~mm}$ e leds de infravermelho, o que permitiu obter imagens noturnas. As imagens foram registradas diariamente, a intervalos de dois minutos, armazenando as informações num banco de dados para posterior análise.

Além do sistema de monitoramento pelas microcâmeras, foi usada a identificação eletrônica dos animais por meio de transponders e antenas estacionárias, possibilitando o registro da freqüência de acesso dos leitões ao abrigo escamoteador e a avaliação da eficiência das ferramentas aplicadas na pesquisa. Todos os animais foram identificados eletronicamente, em todos os tratamentos.

Para a descrição da atividade dos animais e da freqüência de utilização dos locais monitorados, foi realizada análise de regressão entre o número de animais captados pelas antenas e os animais captados pela microcâmera, nesse caso considerada como referência.

\section{RESULTADOS E DISCUSSÃO}

Para os dados referentes à avaliação de possíveis infecções e/ou rejeições ao implante, constatouse que o tratamento que apresentou maior rejeição ao implante do transponder foi a fossa infra-orbital, sendo que em $66 \%$ dos casos houve rejeição logo nos primeiros dias após o implante, caracterizandose pela expulsão do microchip do corpo do animal. A região dos dígitos do pé apresentou resposta intermediária à rejeição, observando-se a expulsão do microchip em 33\% dos animais, ou seja, em apenas um dos três animais. $\mathrm{O}$ tratamento que mostrou melhor desempenho quanto à rejeição foi o implante realizado na cartilagem da base da orelha, sendo também o local de menor sensibilidade no momento da inserção.

Os resultados apresentados na Figura 1 revelaram que entre os diferentes locais de implante dos microchips, aquele que apresentou maior migração, foi a cartilagem da base da orelha, correspondendo a uma distância de migração de $26,9 \mathrm{~mm}$, seguido da fossa infra-orbital, que apresentou um trajeto de migração de $13,1 \mathrm{~mm}$, desde o ponto de inserção até a última verificação no $11^{\mathrm{o}}$ dia. O local onde houve menor movimentação do microchip no corpo do animal foi nos dígitos do pé, onde foi observada migração da ordem de $6 \mathrm{~mm}$. 


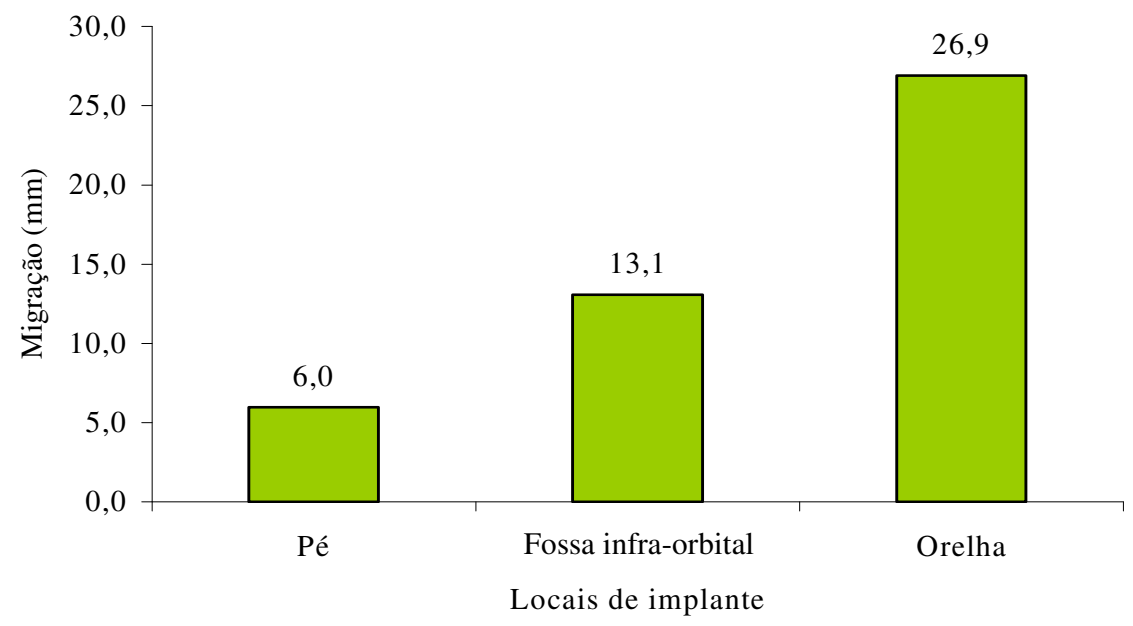

FIGURA 1. Migração dos microchips (mm), em relação aos diferentes locais de aplicação, entre o dia da aplicação e o $11^{\circ}$ dia após o implante.

Outro parâmetro avaliado foi a resposta do animal ao implante dos microchips, com base no ganho de peso diário $(\mathrm{kg})$. Observa-se na Figura 2, reflexo no ganho de peso dos animais submetidos ao implante na região dos dígitos do pé, apresentando ganho de peso médio inferior aos demais de $0,140 \mathrm{~kg} \mathrm{dia}^{-1}$, fato esse que pode ser atribuído à maior sensibilidade do animal no momento do implante. Nesse caso, houve, posteriormente, dificuldade ao caminhar, além de problemas na aplicação, devido ao tipo de tecido encontrado nesse local, o qual dificultava a inserção dos transponders. O índice recomendado para animais nascidos com peso médio de $1.100 \mathrm{~kg}$, é de 0,168 $\mathrm{kg} \mathrm{dia}^{-1}$ para desmame aos 21 dias (MORES et al., 1998). Notou-se, para os animais submetidos ao implante na fossa infra-orbital, GPD $\left(\mathrm{kg} \mathrm{dia}^{-1}\right) 16,7 \%$ inferior ao limite mínimo exigido.

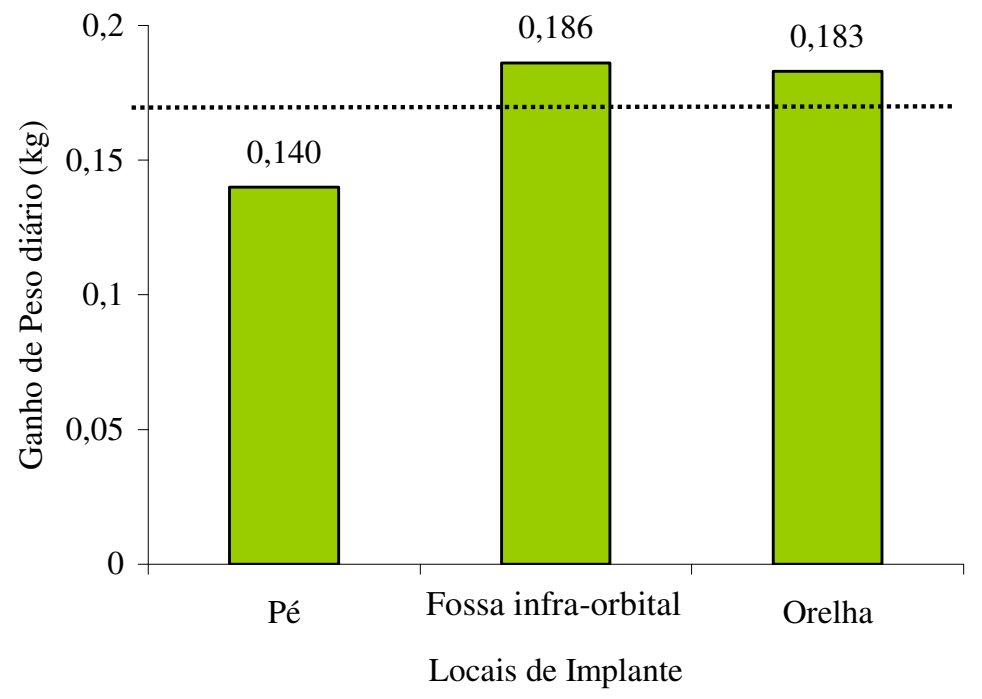

FIGURA 2. Avaliação do ganho de peso diário $(\mathrm{kg})$ dos leitões submetidos aos diferentes locais de implante.

Diante desses resultados, verificou-se que o implante na região da fossa infra-orbital e na cartilagem da base da orelha não apresentaram redução no ganho de peso diário dos animais, que foi de 0,186 e $0,183 \mathrm{~kg} \mathrm{dia}^{-1}$, respectivamente, observando-se ganhos superiores ao recomendado de $10,7 \%$ e $8,9 \%$, respectivamente. 
Com relação às imagens obtidas pelas radiografias, ficou evidente a pequena migração dos transponders na região dos dígitos do pé, apesar de não ser esse o local mais indicado, devido aos problemas de sensibilidade e, conseqüentemente, menor desempenho dos animais submetidos a esse tratamento (Figura 3).
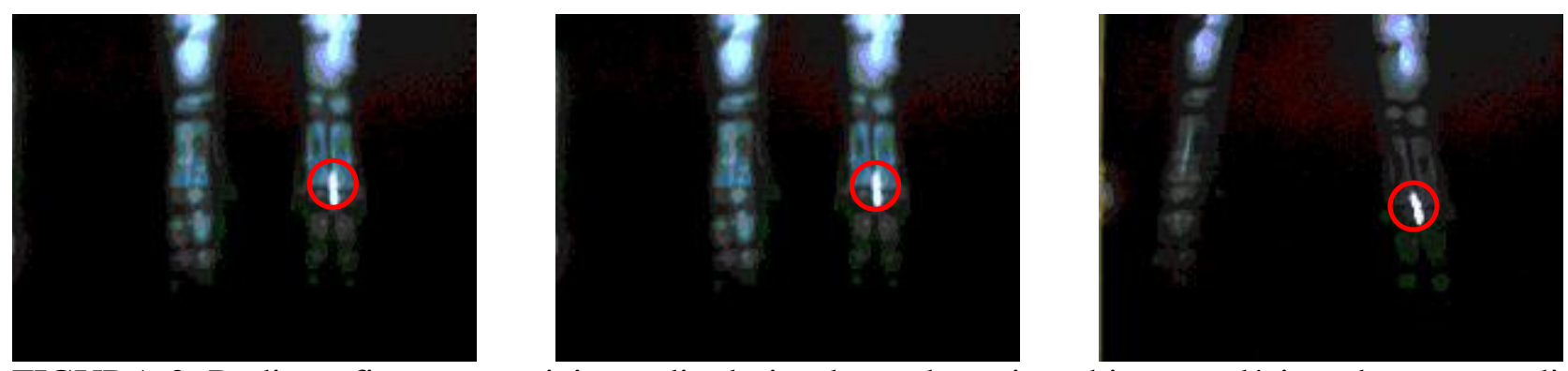

FIGURA 3. Radiografias seqüenciais no dia do implante dos microchips nos dígitos da pata, no dia da operação, no $4^{\underline{0}}$ e no $11^{\mathrm{o}}$ dias, respectivamente.

$\mathrm{Na}$ Figura 4, observa-se o implante na região da cartilagem da base da orelha, verificando-se maior migração do microchip em relação ao seu ponto de inserção. Porém, essa movimentação está dentro do limite aceitável de 60 mm mencionado por KERN (1997), pois ainda se encontra na região próxima à inserção e espera-se que seja localizado sob condições normais por ocasião do abate.
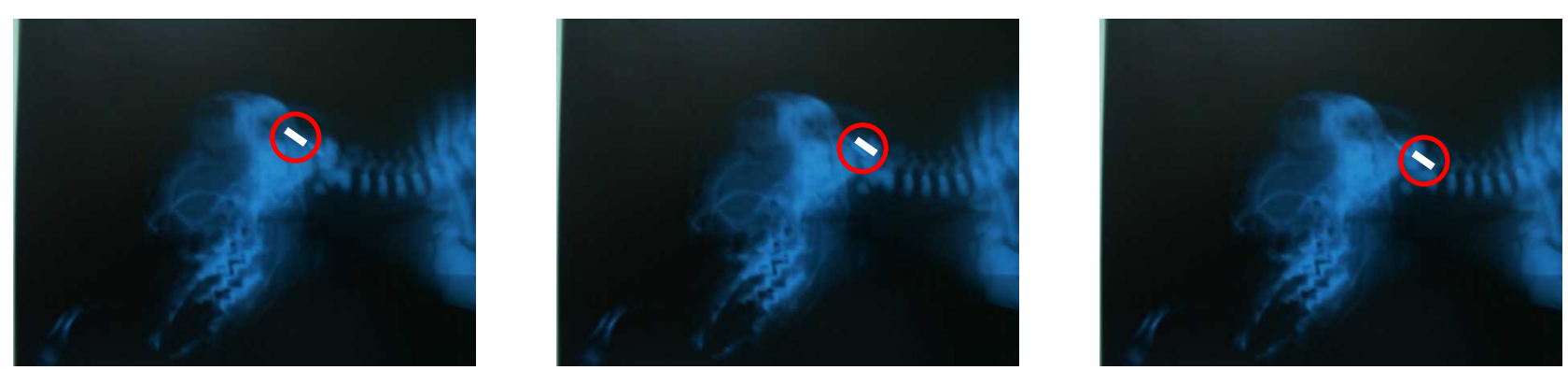

FIGURA 4. Radiografias seqüenciais do implante na cartilagem da base da orelha, no dia da operação, $4^{\circ}$ e $11^{\circ}$ dias, respectivamente.

O movimento migratório do microchip implantado na região da fossa infra-orbital mostrou-se inferior ao da base da orelha; no entanto, foram observados problemas de rejeição, como mencionado anteriormente, não se tratando de um local adequado para o implante do transponder (Figura 5).
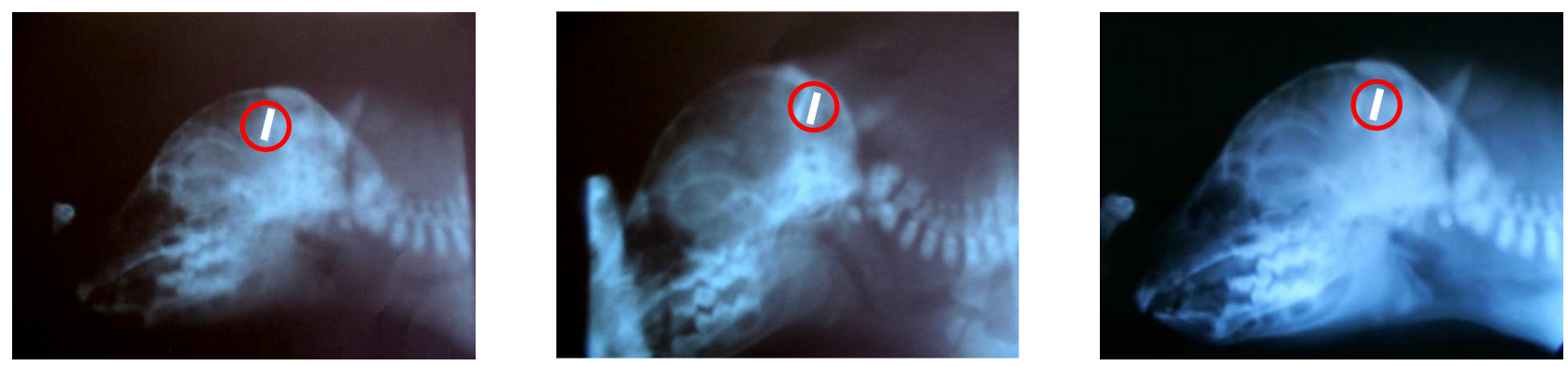

FIGURA 5. Radiografias seqüenciais do implante na região da fossa infra-orbital, no dia da operação, $4^{\circ}$ e $11^{\circ}$ dias, respectivamente. 
Os dados referentes aos sinais captados pelos diferentes sistemas de identificação eletrônica para análise da atividade dos leitões apresentaram variação de acordo com a Figura 6. Por meio dessa comparação entre os sistemas de identificação e a análise de imagem, pode-se verificar que o registro da presença dos leitões no interior dos abrigos ficou abaixo daquele observado na análise de imagem, apresentando menor eficiência para essa aplicação. Talvez a utilização de um controlador de acesso fosse mais adequada ao estudo, visto que as antenas não captaram a identificação de todos os animais no interior do abrigo. Outra possibilidade que evidenciou o menor registro dos sinais emitidos pelos transponders foram as interferências, ocasionadas pela rede de alimentação do sistema de identificação (antenas), a presença de outros equipamentos ligados na rede, estruturas metálicas, etc. Diversas soluções foram propostas na tentativa de evitar essas interferências. Uma delas seria a utilização de um cilindro blindado contendo a unidade receptora (antena) e o aterramento de todo o sistema de identificação (CURTO, 2002).

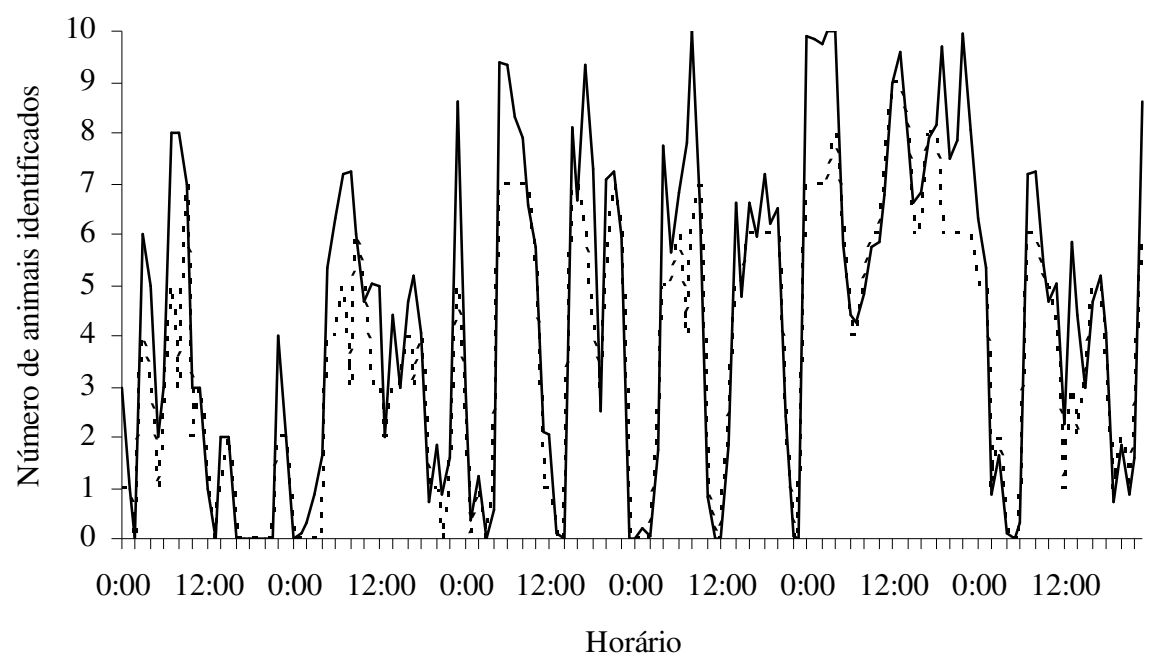

— captados pela câmera - . . . . captado pela antena

FIGURA 6. Variação do número de animais captados pela câmera de vídeo e pelas antenas de recepção do sinal emitido pelos microchips.

Para os dados referentes à validação do sistema de identificação eletrônica, relacionou-se o número de animais captados pelas antenas em função do número de animais captados pelas microcâmeras e utilizou-se de análise de regressão envolvendo dados médios horários de registro. As curvas de ajuste 1:1 apresentaram variação linear, mostrando valor de $R^{2}(0,8677)$ significativo $(\mathrm{P}<0,01)$ pelo teste $\mathrm{F}$, e com nível de confiança de $79 \%$, quando se compararam os dados obtidos pelas antenas em relação às microcâmeras (Figura 7). Dessa forma, a confiabilidade do sistema de análise de imagem foi superior ao uso de transponders, visto que o mesmo apresentou erro na detecção da presença dos animais no interior dos abrigos de $21 \%$. 


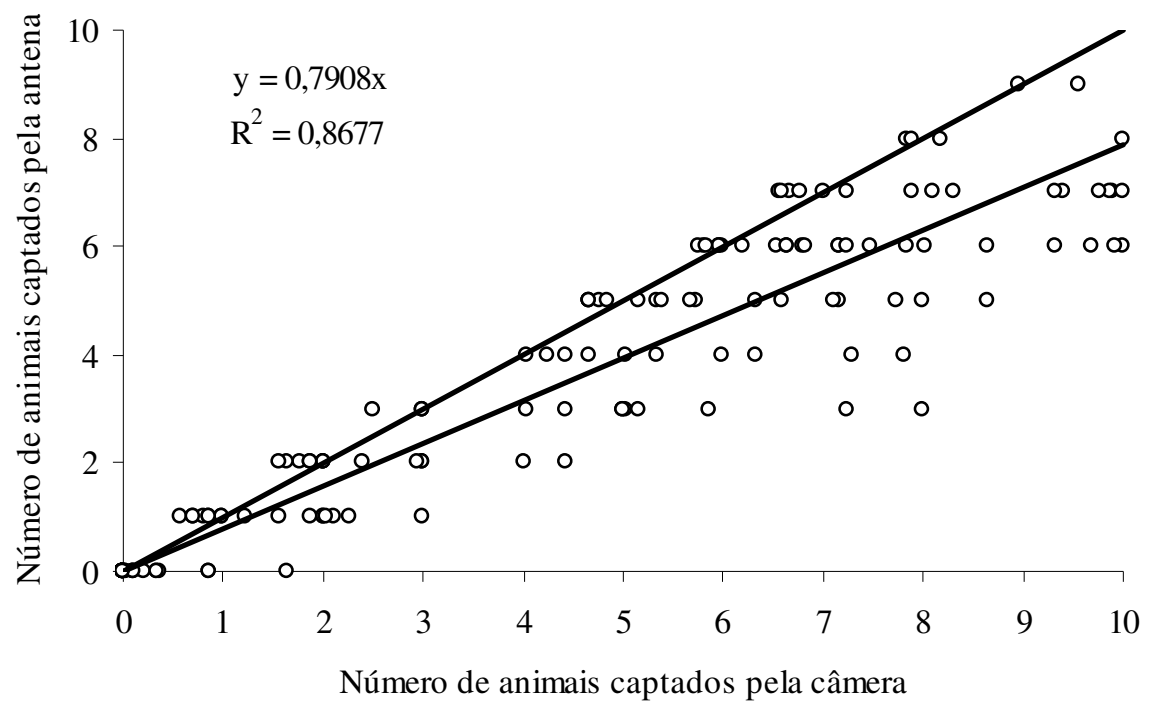

FIGURA 7. Representação da correlação entre registro de dados pelas antenas e pelas microcâmeras.

\section{CONCLUSÕES}

O local mais indicado a receber o implante dos microchips foi a região da cartilagem da base da orelha, por apresentar maior facilidade de aplicação, ótima aceitabilidade pelo animal e mostrar-se dentro de um limite não-crítico de movimentação no corpo do animal.

Quanto às ferramentas tecnológicas, o sistema que se mostrou mais eficiente no estudo do comportamento dos leitões foi a análise de imagem, que pode ser considerada como referência para a validação do sistema de identificação eletrônica por meio de transponders, garantindo o monitoramento em tempo real da atividade dos animais e a freqüência de acesso dos leitões ao abrigo. Já o uso da identificação eletrônica apresentou erro de $21 \%$ na detecção da presença dos animais no interior dos abrigos.

\section{REFERÊNCIAS}

ARNDT, J.; WIEDEMANN, C. Zusammenfassung von Verträglichkeitprüfungen mit Transpondern des elektronischen Markierungssystems INDEXL. Kleintierpraxis, Berlin, v.36, n.1, p.381-9, 1991.

ARTMANN, R. Eletronic identification systems: state of the art and their further development. Computers and Electronics in Agriculture, New York, v.24, n.1, p.5-26, 1999.

CURTO, F.P.F. Estudo do comportamento em matrizes pesadas (frango de corte) em diferentes ambientes utilizando identificação eletrônica e radio-freqüência. 2002. 72 f. Tese (Doutorado em Construções Rurais e Ambiência) - Faculdade de Engenharia Agrícola, Universidade Estadual de Campinas, Campinas, 2002.

CURTO, F.P.F.; MAROCCO, R.; BEHRENS, F.H. Sistemas de identificação eletrônica. Revista do Instituto de Informática da PUCCAMP, Campinas, v.5, n.1, p.165-73, 1997.

KERN, C. Technische leistungsfähigkeit und nutzung von injizierbaren transpondern in der riderhalltung. Dissertation (Technik der Radio Frequenz Identifikation) - Teknisch Universitat München-Weihenstephan, 1997. 
KLINDTWORTH, M.; WENDEL, G.; KLINDTWORTH, K.; PIRKELMANN, H. Eletronic identification of cattle with injectable transponders. Computers and Electronics in Agriculture, New York, v.24, n.1, p.65-79, 1999.

LAMBOOY, E. Das injizieren eines transponders in den tierkörper zur identifikation. In: PETERSEN, B., WELZ, M. (Ed.) Beiträge zur tagung EDV - Anwendung in der herden-und gesundheitskon-trolle. Stuttgart: Eugen Ulmer Verlag, 1990, p.18-22.

LAMBOOY, E.; MERKS, J.W.M. Technique and injection place of eletronic identification numbers in pigs. Zeist: 1989. 335 p. (IVO B-Rapport 335)

MAIBACH, H.I.; ROVEE, D.T. Epidermal wound healing. Chicago: Year Book Medical Publishers, 1991. $422 \mathrm{p}$.

MORES, N.; SOBESTIANSKY, J.; WENTZ, I.; MORENO, A.M. Manejo do leitão do nascimento até o abate. In: SOBESTIANSKY, J.; WENTZ, I.; SILVEIRA, P.R.S.; SESTI, L.A.C. Suinocultura intensiva. Concórdia: Embrapa-CNPSA, 1998. p.135-61.

WISMANS, W.M.G. Identification and registration of animals in the European Union. Computers and Electronics in Agriculture, New York, v.24, n.2, p.99-108, 1999. 